\title{
Die analytische und diagnostische Validität der Bestimmung von Carboxyhämoglobin im Blut und Kohlenmonoxid in der Atemluft von Rauchern und Nichtrauchern
}

\author{
Von G. Heinemann, H. Schievelbein und Fridrun Richter \\ Institut für Klinische Chemie des Deutschen Herzzentrums München des Freistaates Bayern
}

(Eingegangen am 1. September/23. November 1983)

\begin{abstract}
Zusammenfassung: Es wurden die analytische und diagnostische Validität der Bestimmung von Carboxyhämoglobin und Kohlenmonoxid bei Rauchern und Nichtrauchern untersucht. Die beiden Methoden besitzen eine hohe analytische und diagnostische Zuverlässigkeit. Präzision und Richtigkeit erfüllen die an sie gestellten Anforderungen. Der Methodenvergleich ergibt eine gute Korrelation $(\varrho=0,97)$ und eine lineare Regression $\left(y=51,5 \cdot 10^{-3} x+6,26 \cdot 10^{-6}\right)$ im untersuchten Bereich. Die bedingten Wahrscheinlichkeiten der Sensitivität, Spezifität, predictive values und Effizienz erreichen Werte über 0,80 . Unter Berücksichtigung einer standardisierten Probenahme für die Kohlenmonoxid-Bestimmung sind die beiden Methoden von vergleichbarer Validität, wenn sie bei klinischen und epidemiologischen Untersuchungen an Raucher-/Nichtraucher-Kollektiven eingesetzt werden.
\end{abstract}

Analytic and diagnostic validity of the determination of carboxyhaemoglobin in blood, and carbon monoxide in breath of smokers and non-smokers

Summary: The analytic and diagnostic validity of the determination of carboxyhaemoglobin and carbon monoxide in smokers and non-smokers was investigated. Both methods are of high analytic and diagnostic validity. Precision and accuracy fullfill the essential requirements. Comparison of the methods shows good correlation $(\varrho=0.97)$ and linear regression $\left(y=51.5 \cdot 10^{-3} x+6.26 \cdot 10^{-6}\right)$ within the investigated range. Values for the conditional probabilities of sensitivity, specificity, predictive values, and efficiency are higher 0.80 . Taking into account the standardized sample collection for the $\mathrm{CO}$ determination, the two methods are of comparable validity when used in clinical and epidemiological investigations on smokers and non-smokers.

\section{Einfuilhrung}

Die CO-Aufnahme durch inhalierendes Rauchen stellt neben der berufs- und umweltbedingten Kohlenmonoxid (CO)-Exposition eine ganz wesentliche individuelle Belastung des Organismus dar (1). Die physiologische endogene CO-Bildung $(2,3)$ ist äuBerst niedrig und nur in Ausnảhmefällen (1) gering vermehrt. Die Aufnahme von $\mathrm{CO}$ beim Raucher erfolgt ausschließlich über die Lunge, eine Aufnahme über Mund- und Rachenschleimhaut, wie sie für Nikotin bekannt ist, konnte ausgeschlossen werden (4). Infolge der guten Korrelationen von Nikotin- bzw. Cotininkonzentrationen im Blut und Carboxyhämo- globin ( $\mathrm{HbCO}$ )-Werten hat sich die Bestimmung von $\mathrm{HbCO}$ als wertvolle Kenngröße zur Abschätzung der Rauchaufnahme bei Rauchern erwiesen (5). Zunehmendes Interesse gilt der CO-Bestimmung in der Atemluft von Rauchern $(6,7)$.

Wie sich die Häufigkeitsverteilungen physiologisclıer Kenngrößen von Gesunden und Kranken überlappen, so zeigen auch die Histogramme der $\mathrm{HbCO}-$ bzw. CO-Werte von Rauchern und Nichtrauchern diesen Effekt. Dazu tragen bei Rauchern in erster Linie die Rauchgewohnheiten und bei Nichtrauchern die wechselnde CO-Exposition bei. Das 
Festlegen einer Trennlinie zwischen gesundem und krankem Kollektiv mit Hilfe von $\bar{x} \pm 2$ s oder Referenzbereichen hat einige Nachteile, auf die verschiedene Autoren hinweisen (8-11). Daraus ergab sich die Notwendigkeit, auch für die sich überlappenden Verteilungen von Rauchern und Nichtrauchern das Entscheidungskriterium so zu setzen, daß die diagnostischen Zuverlässigkeitskriterien Sensitivität und Spezifität $(12,13,14)$ optimiert und in ihrem Aussagewert durch den predictive value $(15,16)$ und die Effizienz (17) der Methode ergänzt werden.

Zur Ermittlung der Entscheidungsschwelle, die in der Literatur (18) auch als Grenzwert, cut-off point, discrimination value bezeichnet wird, diente die Receiver Operating Characteristic (ROC)-Analyse unter Berechnung des Kurvenpunktes maximalen Informationsgehaltes (I) als Grenzwert (Úbersicht bei 1.c. $(8,13))$. Mittels der Likelihood-Quotienten (LQ)-Geraden (Übersicht bei l.c. (9)) sollten zusätzliche Informationen gewonnen werden. Es stellte sich die Frage, mit welcher analytischen und diagnostischen Zuverlässigkeit HbCO- bzw. CO-Bestimmungen bei Rauchern und Nichtrauchern möglich und ob $\mathrm{CO}$ - und $\mathrm{HbCO}$-Bestimmungen von vergleichbarer Validität sind.

\section{Material und Methoden}

\section{Material}

Die HbCO-Konzentration wurde in 171 EDTA-Blutproben von Nichtrauchern und 472 EDTA-Blutproben von Rauchern, der CO-Gehalt der Atemluft in 87 Atemgasproben von Nichtrauchern und 261 Atemgasproben von Rauchern bestimmt. Für die Regressionsanalyse $\mathrm{HbCO} / \mathrm{CO}$ standen 348 Wertepaare von Nichtrauchern und Rauchern zur Verfügung. Die Zuordnung der Probanden zur Rauchergruppe erfolgte mittels eines Fragebogens mit genauen Angaben zum Rauchverhalten vor der Probengewinnung (z. B. Konsum und Zeitpunkt während der vergangenen Tage, der letzten 24 Stunden, Zeitpunkt der letzten Zigarette, Inhalation ja/nein, Inhalationstiefe). In die Rauchergruppe wurden nur inhalierende Raucher, die bis etwa $10 \mathrm{~min}$ vor der Probengewinnung ihre Rauchgewohnheiten beibehalten hatten, aufgenommen. Die Blut- und kurz darauf die Atemgasproben wurden am späten Nachmittag gewonnen.

Geräte

CO-Oximeter IL 282 (Instrumentation Laboratory, Inc., Hersel); Infrarot-CO-Analyzer 867 (Beckman Instruments, München); PVC-Plastikbeutel mit verschließbarem Mundstück, Inhalt 9,4 1 (Fa. R. Steindl, München);

EDTA-Gefäße, $4 \mathrm{ml}$ (Fa. Greiner, Nürtingen);

Kalibriergase 0; 50 und $80 \times 10^{-6} \mathrm{CO}$ (Fa. Linde, München).

Methoden

Für die Atemgas-Probengewinnung wurden in einem Vorversuch die Dichtheit der Plastikbeutel für CO geprüft und die folgende standardisierte Atemtechnik aus drei Atemtechnik-Varianten ausgewählt:
1. Tiefes Einatmen,

2. $10 \mathrm{~s}$ Luft anhalten,

3. normal ausatmen,

4. maximales Ausatmen (exspiratorisches Reservevolumen) in den Plastikbeutel,

5. normal atmen während $10 \mathrm{~s}$,

6. Wiederholen von 1.-5., bis der Beutel gefüllt ist.

$\mathrm{HbCO}$ wurde mit dem CO-Oximeter IL 282 und der CO-Gehalt der Atemluft mit dem Infrarot-Analyzer 867 bestimmt. Die statistischen Berechnungen (19) erfolgten auf dem Tischrechner 9845 B (Hewlett u. Packard, München) mit den Programmen „Regression Analysis" und "General Statistics".

\section{Ergebnisse}

\section{Analytische Zuverlässigkeit}

Zur Überprüfung der Repetierbarkeit des CO-Oximeters IL 282 wurde $\mathrm{HbCO}$ in einer Blutprobe 14 mal bestimmt. Bei einem Mittelwert von $\bar{x}=37,5$ $\times 10^{-3} \mathrm{HbCO}$ mit einer Standardabweichung $\pm \mathrm{s}=$ $0,75 \times 10^{-3}$ ergab sich ein Variabilitäts=Koeffizient (VK) von 0,02 . Die Richtigkeit lag zwischen 0,005-0,01 Abweichung. Für das IR-Spektrometer wurden mit $11 \mathrm{CO}$-Bestimmungen aus einem Atemgasbeutel folgende Werte ermittelt: $\bar{x}=47,9 \times$ $10^{-6}, \pm \mathrm{s}=0,27 \times 10^{-6}, \mathrm{VK}=0,0056$. Die Richtigkeit lag 0,01 über dem Sollwert von $50 \times 10^{-6} \mathrm{CO}$. Im Methodenvergleich ließ sich für die $\mathrm{n}=348$ Wertepaare ein Rangkorrelationskoeffizient (Spearman) von $\varrho=0,97$ errechnen. Die Gleichung der Regressionsgeraden war $y=51,5 \cdot 10^{-3} x+6,26$. $10^{-6}$ (Abb. 1).

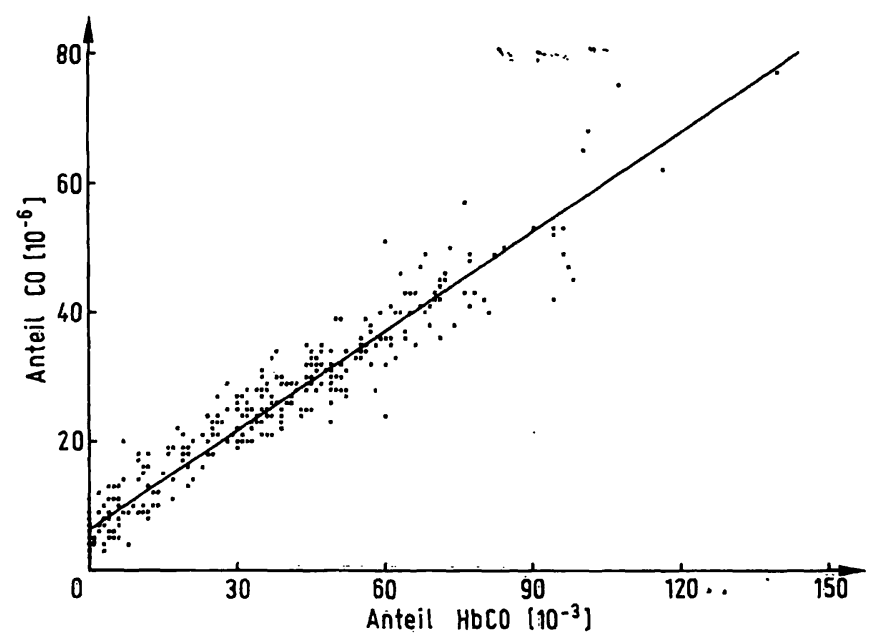

Abb. 1. Korrelation der Bestimmungen von $\mathrm{HbCO}$ und $\mathrm{CO} n=$ $348, \varrho=0,97$ (Spearman), Regressionsgerade: $y=51,5$ $\cdot 10^{-3} x+6,26 \cdot 10^{-6}$.

\section{ROC-Analyse und LQ-Geraden}

Für die Häufigkeitsverteilungen wurden die $\mathrm{HbCO}-$ Werte $\left(10^{-3}\right)$ nach $\log \left(10^{3} x+1,5\right)$, die CO-Werte $\left(10^{-6}\right)$ nach $\log \left(10^{6} x+1,5\right)$ transformiert. Wie in Abbildung 2, so ist auch in Abbildung 3 eine Uberlappung der beiden Verteilungen vorhanden. Die 


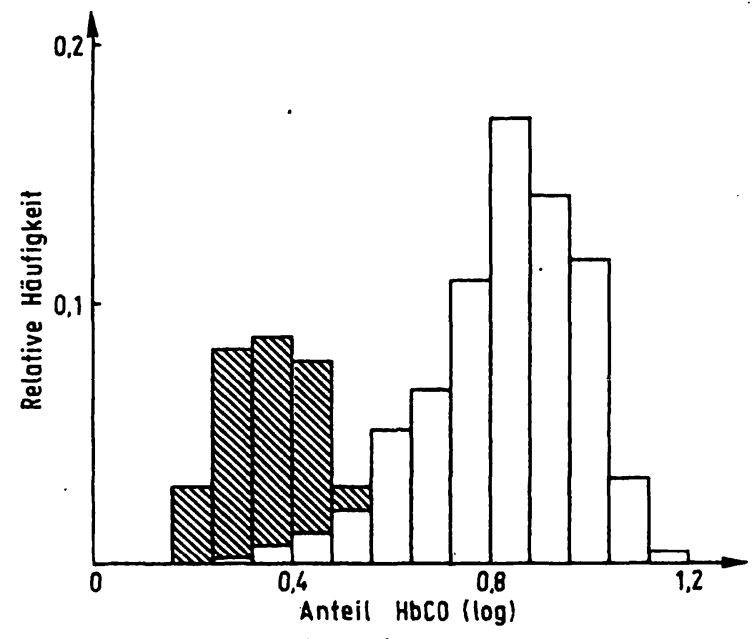

Abb. 2. Häufigkeitsverteilung der nach $\log \left(10^{3} x+1,5\right)$ transformierten HbCO-Werte der Raucher und Nichtraucher (= an).

statistischen Maßzahlen der Häufigkeitsverteilungen wurden rücktransformiert und sind in Tabelle $1 \mathrm{zu}$ sammengefaßt. Für die ROC-Analyse und die Berechnung der LQ wurden die gleichen Klassenbreiten wie in Abbildung 2 bzw. 3 verwendet.

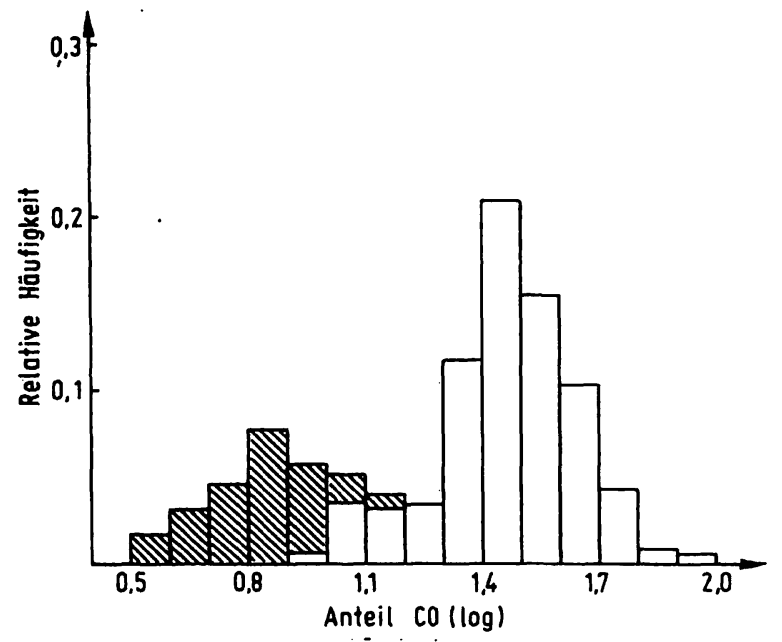

Abb. 3. Häufigkeitsverteilung der nach $\log \left(10^{6} x+1,5\right)$ transformierten CO-Werte der Raucher und Nichtraucher (= (N).

In Tabelle 2 sind die Daten der Entscheidungsmatrix enthalten, die sich bei einer schrittweisen Verschiebung der Entscheidungsschwelle im Ưberlappungsbereich ergeben. Tabelle 3 enthält die Fraktile für die Kenngrößen der diagnostischen Zuverlässigkeit

Tab. 1. Statistische Maßzahlen (Antilog des Medians $\tilde{x}$, des 10. und des 90. Perzentils ( $\left.P_{10}, P_{90}\right)$ ) der Häufigkeitsverteilungen in Abbildung 2 und 3.

\begin{tabular}{|c|c|c|c|c|c|c|c|}
\hline & \multicolumn{3}{|c|}{ Anteil $\mathrm{HbCO}\left(10^{-3}\right)$} & & \multicolumn{3}{|c|}{ Anteil CO $\left(10^{-6}\right)$} \\
\hline & $\overline{\mathbf{x}}$ & $P_{10}$ & $P_{90}$ & & $\overline{\mathbf{x}}$ & $P_{10}$ & $\mathrm{P}_{90}$ \\
\hline $\begin{array}{l}\text { Nichtraucher } n=171 \\
\text { Raucher } n=472\end{array}$ & $\begin{array}{l}22 \\
69\end{array}$ & $\begin{array}{l}17 \\
40\end{array}$ & $\begin{array}{r}27 \\
102\end{array}$ & $\begin{array}{l}\text { Nichtraucher } n=87 \\
\text { Raucher } n=261\end{array}$ & $\begin{array}{r}6,5 \\
29,5\end{array}$ & $\begin{array}{r}4,5 \\
15,5\end{array}$ & $\begin{array}{r}9,5 \\
44,5\end{array}$ \\
\hline
\end{tabular}

Tab. 2. Entscheidungsmatrices für die Verteilungen der HbCO- und CO-Werte bei verschiedenen Schwellen (log Werte). $\mathrm{TP}=$ richtig positiv; $\mathrm{FP}=$ falsch positiv; $\mathrm{TN}=$ richtig negativ; $\mathrm{FN}=$ falsch negativ.

\begin{tabular}{|c|c|c|c|c|c|c|c|c|c|c|}
\hline & \multicolumn{5}{|c|}{$\begin{array}{c}\text { HbCO } \\
\text { Schwelle bei }\end{array}$} & \multicolumn{5}{|c|}{$\begin{array}{c}\mathrm{CO} \\
\text { Schwelle bei }\end{array}$} \\
\hline & 0,24 & 0,32 & 0,40 & 0,48 & 0,56 & 0,89 & 0,99 & 1,09 & 1,19 & 1,29 \\
\hline TP & 472 & 471 & 467 & 460 & 446 & 261 & 259 & 247 & 236 & 224 \\
\hline FN & 0 & 1 & 5 & 12 & 26 & 0 & 2 & 14 & 25 & 37 \\
\hline TN & 19 & 71 & 123 & 166 & 171 & 60 & 78 & 84 & 87 & 87 \\
\hline FP & 152 & 100 & 48 & 5 & 0 & 27 & 9 & 3 & 0 & 0 \\
\hline
\end{tabular}

Tab. 3. Fraktile für die Kenngrößen der diagnostisehen Zuverlässigkeit der HbCO- und CO-Bestimmung bei verschiedenen Schwellen (log Werte).

\begin{tabular}{llllll}
\hline & Schwelle bei & Sensitivität & Unsensitivität & Spezifität & Unspezifität \\
\hline \multirow{4}{*}{ HbCO } & 0,24 & 1 & 0 & 0,1111 & 0,8888 \\
& 0,32 & 0,9978 & 0,0021 & 0,4152 & 0,5847 \\
& 0,40 & 0,9894 & 0,0105 & 0,7192 & 0,2807 \\
& 0,48 & 0,9745 & 0,0254 & 0,9707 & 0.0292 \\
CO & 0,56 & 0,9449 & 0,0550 & 1 & 0 \\
& 0,89 & 1 & 0 & 0,6896 & 0,3103 \\
& 0,99 & 0,9923 & 0,0076 & 0,8965 & 0,103 \\
& 1,09 & 0,9463 & 0,0536 & 0,9655 & 0,0344 \\
\hline
\end{tabular}


bei den verschiedenen Schwellen. In Abbildung 4 sind die ROC-Kurven unter Angabe des Punktes mit höchstem Informationsgehalt (I-Wert) für die in den Kollektiven vorhandene Prävalenz der Raucher dargestellt. Für I $=0,6993$ bit wurde ein Grenzwert von $16 \times 10^{-3} \mathrm{HbCO}$ und für $\mathrm{I}=0,6062$ bit ein Grenzwert von $8,4 \times 10^{-6} \mathrm{CO}$ errechnet. Für die in der Bevölkerung vorkommenden niedrigeren Prävalenzen (Raucher $\mathbf{P}(\mathbf{k})=0,43$, Raucherinnen $\mathrm{P}(\mathbf{k})=$ 0,27 , Raucher und Raucherinnen $P(k)=0,35$ ) erhöhten sich die Grenzwerte auf $21 \times 10^{-3} \mathrm{HbCO}$ und $14,3 \times 10^{-6} \mathrm{CO}$.

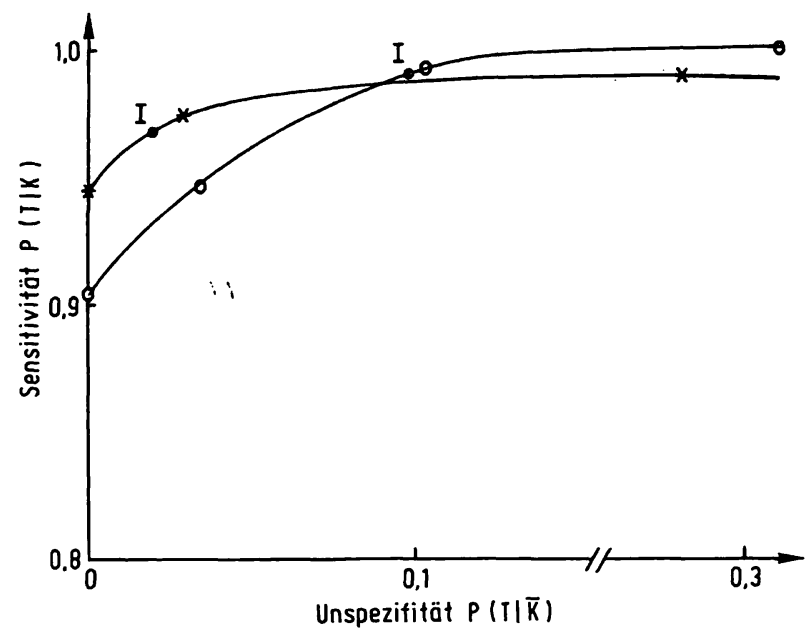

Abb. 4. ROC-Kurven für die $\mathrm{HbCO}-(\star-\star)$ und $\mathrm{CO}-\mathrm{Me}-$ thode.

T: Test positiv, $\mathrm{K}=$ Raucher, $\overline{\mathrm{K}}=$ Nichtraucher, $\mathrm{I}=$ Punkt mit max. Information für $\mathrm{P}(\mathrm{k})=0,73(\mathrm{HbCO})$ und $P(k)=0,75(\mathrm{CO})$.

Zur Berechnung der diskreten fraktilierten LQ wurden nur die Klassen im Überlappungsbereich verwendet. Die LQ für die Anteile im Bereich der
Úberlappung der HbCO- bzw. CO-Bestimmung sind in Tabelle 4 aufgelistet. Daraus ergeben sich die in Abbildung 5 dargestellten LQ-Geraden (Risikogeraden) für den positiven und negativen Test. Die Validitätsquotienten (VQ) für die $\mathrm{HbCO}$ - und die CO-Bestimmung sind 866 und 1066.
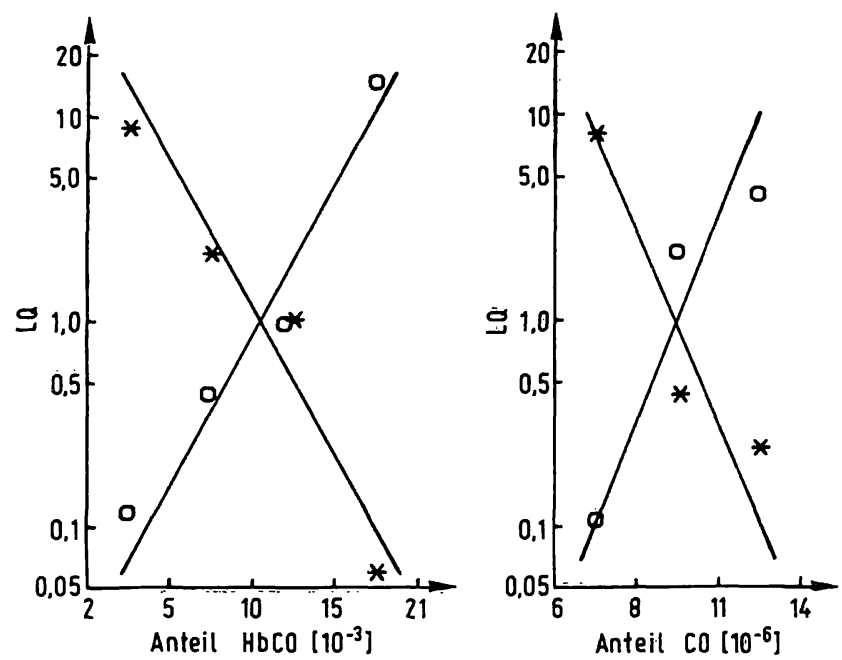

Abb. 5. Positive (O-O) und negative LQ-Geraden für die $\mathrm{HbCO}$ - und CO-Bestimmung.

\section{Kenngrößen der diagnostischen Validität}

Die bedingten Wahrscheinlichkeiten von Sensitivität und Spezifität für die Grenzwerte $16 \times 10^{-3}$ bzw. 21 $\times 10^{-3} \mathrm{HbCO}$ und $8,4 \times 10^{-6}$ bzw. $14,3 \times 10^{-6} \mathrm{CO}$ zeigt Tabelle 5. Die unter Anwendung des Bayesschen Theorems $(15,20)$ berechneten predictive values für die unterschiedlichen Prävalenzen der Kollektive und der Bevölkerung sind unter B in Tabelle 5 aufgeführt. Unter $\mathrm{C}$ wird die Effizienz̄ der Methoden angegeben.

Tab. 4. Likelihood-Quotienten $L Q_{i}$ bzw. $L Q_{i}$ für die Úberlappungsbereiche der log-transformierten HbCO- und CO-Werte. $\mathrm{D}=$ Raucher, $\overline{\mathrm{D}}=$ Nichtraucher

\begin{tabular}{|c|c|c|c|c|c|c|}
\hline Klasse & $N_{D}$ & $N_{\bar{D}}$ & $\mathrm{frD}_{i}$ & $\operatorname{frD}_{i}$ & $L^{L} Q_{i}$ & $\mathbf{L} \bar{Q}_{i}$ \\
\hline & & & & $\mathrm{HbCO}$ & & \\
\hline $0,24-0,32$ & 1 & 52 & 0,0384 & 0,3421 & 0,1124 & 8,8949 \\
\hline $0,32-0,40$ & 4 & 52 & 0,1538 & 0,3421 & 0,4496 & 2,2237 \\
\hline $0,40-0,48$ & 7 & 43 & 0,2692 & 0,2828 & 0,9516 & 1,0508 \\
\hline \multirow[t]{2}{*}{$0,48-0,56$} & 14 & 5 & 0,5384 & 0,0328 & 16,369 & 0,0610 \\
\hline & $\Sigma 26$ & $\Sigma 152$ & & & & \\
\hline ' & & & & $\mathrm{CO}$ & & \\
\hline $0,89-0,99$ & 2 & 18 & 0,08 & 0,666 & $0.1 \overline{2} 0$ & 8,330 \\
\hline $0,99-1,09$ & 12 & 6 & 0,48 & 0,222 & 2,160 & 0,462 \\
\hline \multirow[t]{2}{*}{$1,09-1,19$} & 11 & 3 & 0,44 & 0,111 & 3,960 & 0,252 \\
\hline & $\Sigma 25$ & $\Sigma 27$ & & & $\therefore$ & \\
\hline
\end{tabular}


Tab. 5. Bedingte Wahrscheinlichkeiten bei verschiedenen Grenzwerten (G) der HbCO- und CO-Bestimmung für: A: Sensitivität und Spezifität; B: predictive values für positiven $(T)$ und negativen $(T)$ Test unter Vorgabe verschiedener Prävalenzen $P(k)$; $C$ : Effizienz.

\begin{tabular}{|c|c|c|c|c|c|c|c|c|c|}
\hline & \multirow[b]{2}{*}{ Kenngröße } & \multicolumn{4}{|c|}{$\mathrm{HbCO}$} & \multicolumn{4}{|c|}{$\mathrm{CO}$} \\
\hline & & \multicolumn{2}{|c|}{$G=16 \times 10^{-3}$} & \multicolumn{2}{|c|}{$G=21 \times 10^{-3}$} & \multicolumn{2}{|c|}{$G=8,4 \times 10^{-6}$} & \multicolumn{2}{|c|}{$G=14,3 \times 10^{-6}$} \\
\hline A & $\begin{array}{l}\text { Sensitivität } \\
\text { Spezifität }\end{array}$ & & $\begin{array}{l}0,96 \\
0,97\end{array}$ & & $\begin{array}{l}0,94 \\
1\end{array}$ & & $\begin{array}{l}0,99 \\
0,89\end{array}$ & & $\begin{array}{l}0,88 \\
1\end{array}$ \\
\hline B & $\begin{array}{l}\text { predictive values für } \\
\begin{aligned} \mathrm{P}(\mathrm{k}) & =0,73 \\
& =0,43 \\
& =0,35 \\
& =0,27\end{aligned}\end{array}$ & $\begin{array}{l}T \\
0,98 \\
0,96 \\
0,94 \\
0,92\end{array}$ & $\begin{array}{l}\bar{T} \\
0,89 \\
0,96 \\
0,97 \\
0,98\end{array}$ & $\begin{array}{l}\mathbf{T} \\
1 \\
1 \\
1 \\
1\end{array}$ & $\begin{array}{l}\bar{T} \\
0,86 \\
0,95 \\
0,96 \\
0,97\end{array}$ & $\begin{array}{l}\mathrm{T} \\
0,96^{*} \\
0,87 \\
0,82 \\
0,76\end{array}$ & $\begin{array}{l}\bar{T} \\
0,96^{*} \\
0,99 \\
0,99 \\
0,99\end{array}$ & $\begin{array}{l}\mathrm{T} \\
1^{*} \\
1 \\
1 \\
1\end{array}$ & $\begin{array}{l}\overline{\mathrm{T}} \\
0,73^{*} \\
0,91 \\
0,93 \\
0,95\end{array}$ \\
\hline C & Effizienz & & 0,96 & & 0,95 & & 0,96 & & 0,91 \\
\hline
\end{tabular}

$* P(k)=0,75$

\section{Diskussion}

Für die Bestimmung von $\mathrm{HbCO}$ und $\mathrm{CO}$ gibt es klinische und epidemiologische Gründe, so z.B. im Rahmen von Programmen zur Behandlung von $\mathrm{Pa}-$ tienten mit dem Risikofaktor „Rauchen“ oder bei Untersuchungen zum Einfluß von ZigarettenrauchBestandteilen auf den Organismus. Um Raucher und Nichtraucher zu differenzieren, bedarf es einer Indikatorsubstanz, die nach Saloojee et al. (21) im Idealfall vier Anforderungen erfüllen sollte:

1. Spezifität für Tabakrauch,

2. Unabhängigkeit von genetischen, ernährungsund umweltbedingten Einflüssen,

3. lange Halbwertszeit,

4. leichte und zuverlässige Bestimmung.

Aber weder $\mathrm{HbCO}$ oder $\mathrm{CO}$ noch eine der anderen möglichen Kenngrößen wie Nikotin, Cotinin und Thiocyanat (SCN) im Blut entsprechen den 4 Anforderungen gleichermaßen.

Zur Messung der Rauchaufnahme werden üblicherweise Nikotin, Cotinin und SCN in Körperflüssigkeiten sowie $\mathrm{HbCO}$ im Blut und $\mathrm{CO}$ in der Ausatmungsluft herangezogen. Die meist gaschromatographisch durchgeführten Bestimmungen von Nikotin und Cotinin in Blut und/oder Harn sind zeit- und personalintensiv. Hingegen lassen sich $\mathrm{SCN}$ und $\mathrm{HbCO}$ einfacher und schneller bestimmen, vor allem, wenn mechanisierte Methoden verwendet werden. Besonders einfach gestaltet sich die infrarotspektrometrische CO-Bestimmung in der Atemluft, zumal die Probengewinnung nicht-invasiv erfolgt.

Die Wahl der jeweiligen Kenngröße hängt wesentlich von der Fragestellung ab (z.B., ob Kurz- oder Langzeitänderungen der Rauchaufnahme untersucht werden sollen). Als Halbwertszeiten gelten für Nikotin 20-50 min $(22,23)$, für $\mathrm{HbCO}$ bei geringer körperlicher Aktivität $4 \mathrm{~h}$ (24), für Cotinin 10-30 h $(23,25)$ und für Thiocyanat $14 \mathrm{~d}(26)$. Die Aufnahme von Nikotin und Cyanid erfolgt sowohl über die Mund- und Darmschleimhäute als auch über die Lungen, die von $\mathrm{CO}$, wie bereits einleitend erwähnt, allein über die Alveolen. Cotinin als Hauptmetabolit des Nikotins entsteht vorwiegend in der Leber.

Eine Einschränkung der Spezifität von SCN, $\mathrm{HbCO}$ und $\mathrm{CO}$ im Exhalat kann sich durch Umwelteinflüsse, Nahrungsmittel und Medikamente ergeben (21). Auf die sehr niedrige endogene $\mathrm{HbCO}$-Bildung wurde hingewiesen, der endogene SCN-Anteil ist ebenfalls minimal. Im Hinblick auf die Spezifität ist Cotinin vor Nikotin einzureihen, gefolgt von Thiocyanat und Carboxyhämoglobin, die eine vergleichbare Aussagekraft (21) haben. Der Stellenwert der COBestimmung in der Atemluft wird im Vergleich zum $\mathrm{HbCO}$ in der vorliegenden Studie dargelegt.

Die Ergebnisse zeigen, daß Präzision, Richtigkeit und Variabilitätskoeffizient der Bestimmung von $\mathrm{HbCO}$ und $\mathrm{CO}$ die Anforderungen an die analytische Zuverlässigkeit erfüllen. Auf die Notwendigkeit einer standardisierten Probenahme für CO-Bestimmungen in der Atemluft weisen Stewart (27), Jones (28) und Rawbone (29) hin. Diese Empfehlung ist besonders zu beachten, da Präzision und Richtigkeit die diagnostische Zuverlässigkeit beeinflussen (30-33). So nehmen bei Zunahme der Streuung Sensitivität und predictive value deutlich $a b$, ändert sich die Spezifität hingegen nur wenig und beeinflussen Änderungen der Richtigkeit die UUberlappung zweier Kollektive. Die Probenahme für die HbCOBestimmung erfolgt in üblicher Weise und arterielles 
wie venöses EDTA-Blut ergeben die gleichen HbCO-Werte (29). Die gute Korrelation der Wertepaare und die Linearität der Geraden in Abbildung 1 entsprechen den Angaben anderer Autoren $(7,34$, 35). Bei Werten über $140 \times 10^{-3} \mathrm{HbCO}$ und $90 \times$ $10^{-6} \mathrm{CO}$ weicht die Regressionsgerade exponentiell von der Linearität ab (36).

Für die ROC-Analyse wurden die rechtsschiefen Verteilungen der $\mathrm{HbCO}$ - und $\mathrm{CO}-$ Werte mittels logTransformation normalisiert, um die Voraussetzungen $(8,32,37)$ bezüglich Verteilung und Varianz zu erfüllen. Unter dem Einfluß der hohen Prävalenzen $P(k) \cong 0,7$ der Raucher in den untersuchten Kollektiven ergaben sich für die $\mathrm{HbCO}$ - bzw. CO-Bestimmung als optimierte Grenzwerte zwischen Rauchern und Nichtrauchern $16 \times 10^{-3} \mathrm{HbCO}$ und $8,4 \times 10^{-6}$ CO. Diese Diskriminanzschwellen stimmen mit den Angaben von Saloojee et al. (21) und Vogt et al. (24) überein, die allerdings Mittelwert und 0,95-Bereich als Schwellen verwendeten. Die in der Bevölkerung vorkommenden niedrigeren Prävalenzen bewirkten als Folge der Verschiebung von I auf der ROC-Kurve eine Erhöhung der Grenzwerte auf $21 \times 10^{-3}$ $\mathrm{HbCO}$ und $14,3 \times 10^{-6} \mathrm{CO}$. Hinweise auf den allgemeinen Zusammenhang derartiger Grenzwertänderungen als Auswirkung des sog. Verdünnungseffektes finden sich bei Büttner (8), Vecchio (15), Oellerich \& Haeckel (38). Sensitivität und Spezifität wie auch die predictive values des positiven und negativen Tests unterliegen dem Einfluß der Prävalenzen, der aber bei beiden Methoden nur unwesentlich bemerkbar wird. Der hohe richtig-positive und der zugleich niedrige falsch-positive Anteil, die den gering unterschiedlichen Verlauf der beiden ROC-Kurven bestimmen, sprechen für eine gleich hohe diagnostische Validität der beiden Methoden. Ähnliche ROC-Kurven fanden sich in der Literatur nur vereinzelt, wie z.B. bei der Bestimmung der Kreatinkinase $(\mathrm{CK})$ und CK-MB $(33,39)$ und für die Harnsäurebestimmung bei schwerer Gestose (40):

Die Anwendung der ROC-Analyse ist infolge der bereits erwähnten Voraussetzungen begrenzt. Hinzu kommt der Informationsverlust durch die Grenzwertbildung mit ihrer Ja/Nein-Aussage $(41,42)$ und die fehlende Möglichkeit, die Quantität des Resultates zu berücksichtigen. Dennoch stellt die ROC-
Analyse zusammen mit der Anwendung des Bayes'schen Theorems eine wesentliche Hilfe bei der Beurteilung der diagnostischen Validität dar, da der Informationsgewinn mit einfachen Rechenhilfsmitteln möglich ist und bei Feld- und Screeninguntersuchungen die niedrigen Prävalenzen berücksichtigt werden können.

Bei der Validitätsbeurteilung mittels der LQ-Geraden entfallen die bei der ROC-Analyse bestehenden Nachteile. Vielmehr kann unter Berücksichtigung der Resultatquantität für jedes einzelne Testresultat ein Informationsgewinn erzielt werden, der die Frage beantwortet, wie hoch/gering die „Chance“" (Risiko, Wahrscheinlichkeit) für das Vorliegen einer vermuteten Krankheit oder Zustandes ist. Bezogen auf unser Raucherkollektiv läßt sich aus der LQ-Geraden (Risikogeraden), z. B. für den positiven Test, die Chance der Zugehörigkeit zum Kollektiv „Raucher“ ermitteln. Die Zunahme der HbCO-Konzentration von $4,9 \times 10^{-3}$ auf $10,5 \times 10^{-3}$ bedeutet eine $10 \mathrm{fa}-$ che Steigerung des Riskos, als Raucher eingestuft zu werden. Die gleiche Risikozunahme ergibt sich bei einem Anstieg der CO-Konzentration von $7 \times 10^{-6}$ auf $9,5 \times 10^{-6}$.

Eine weitere Information zur Validität der beiden Bestimmungsmethoden liefert der VQ (9). Ein hoher VQ spricht für eine hohe Validität einer Methode. Sowohl die Bestimmung von $\mathrm{CO}(\mathrm{VQ}=1066)$ wie auch diejenige von $\mathrm{HbCO}(\mathrm{VQ}=866)$ lassen sich als Methoden mit hoher Validität beurteilen, wenn zum Vergleich die VQ-Werte anderer Methoden (9) hinzugezogen werden. 'Der Validitätsunterschied beider Methoden ist gering.

Die Untersuchung zeigt, daß die $\mathrm{HbCO}$ - und $\mathrm{CO}$ Bestimmung eine hohe diagnostische und analytische Zuverlässigkeit bei Rauchern und Nichtrauchern besitzen, eine hohe Effizienz aufweisen und von vergleichbarer Validität sind.

\section{Danksagung}

Für die technische Assistenz danke ich Fräulein K. Löschenkohl und Fräulein $I$. Köster. 


\section{Literatur}

1. Weir, F. W. \& Fabiano, B. S. (1982) J. Occup. Med. 24, 519-525.

2. Coburn, R. F., Williams, W. J., White, P. \& Kahn, S. B. (1967) J. Clin. Invest. 46, 346-356.

3. Sjostrand, T. (1949) Scand. J. Clin. Lab. Invest. 1, 201-214.

4. Wald, N. I., Idle, M. \& Bailey, A. (1978) Thorax 33, 201-206.

5. Ashton, H., Stepney, R. \& Thompson, J. W. (1981) Brit. Med. J. 282, 10-13.

6. Jarvis, M. J., Russell, M. A. H. \& Saloojee, Y. (1980) Brit. Med. J. 281, 484-485.

7. Wald, N. J., Idle, M. Boreham, J. \& Bailey, A. (1981) Thorax 36, 366-369.

8. Büttner, J. (1977) J. Clin. Chem. Clin. Biochem. 15, 1-12.

9. Keller, H. \& Gessner, U. (1981) Med. Lab. 34, 3-39.

10. Galen, R. S. \& Gambino, S. R. (1979) Norm und Normabweichung klinischer Daten, Fischer Verlag, Stuttgart, New York.

11. Gross, R. \& Wichmann, H. E. (1979) Med. Welt 30, 2-14.

12. Remein, Q. R. \& Wilkerson, H. L. C. (1961) J. Chronic Dis. $13,6-21$.

13. Lusted, L. B. (1968) Introduction to medical decision making, C. C. Thomas Publ. Springfield, Ill.

14. McNeil, B. J., Keeler, E. \& Adelstein, S. J. (1975) N. Engl. J. Med. 293, 211-215.

15. Vecchio, Th. J. (1966) N. Engl. J. Med. 274, 1171-1173.

16. Archer, Ph. G. (1978) Am. J. Clin. Pathol. 69, 32-35.

17. Galen, R. S. (1980) Pediatr. Clin. North Am. 27, 861-869.

18. Sunderman, F. W. (1975) Clin. Chem. 21, 1873-1877.

19. Sachs, H. (1978) Angewandte Statistik, 5. Aufl. 43-45, Verlag Springer, Berlin.

20. Barnoon, S. \& Wolfe, H. (1972) Measuring the effectiveness of medical decisions, C. C. Thomas, Springfield, Ill.

21. Saloojee, Y., Vesey, C. J., Cole, P. V. \& Russell, M. A. H. (1982) Thorax 37, 521-525.

22. Isaac, P. F. \& Rand, M. J. (1972) Nature 236, 308-310.

23. Kyerematen, G. A., Damiano, M. E., Dvorchik, B. H. \& Vesell, E. S. (1982) Clin. Pharm. Ther. 32, 769-780.
24. Vogt, Th. M., Selvin, St., Widdowson, G. \& Hulley, St. B. (1977) Am. J. Public Health 67, 545-549.

25. Langone, J. J., Gjika, H. W. \& van Vunakis, H. (1973) Biochemistry $12,5025-5030$.

26. Pettigrew, A. R. \& Fell, G. S. (1972) Clin. Chem. 18, 996-1000.

27. Stewart, R. D. (1974) Essays in Toxicology (Hayes, W., ed.), Academic Press, New York.

28. Jones, R. H., Ellicott, M. F., Cadigan, J. B. \& Gaensler, E. A. (1958) J. Lab. Clin. Med. 51, 553-564.

29. Rawbone, R. G., Coppin, C. A. \& Guz, A. (1976) Clin. Sci Mol. Med. 51, 495-501.

30. Büttner, H. (1980) Validität klinisch-chemischer Befunde (Lang, H., Rick, W. \& Büttner, H., eds.), Springer Verlag, Berlin, Heidelberg, New York.

31. Martin, H. F., Gudzinowicz, B. \& Fanger, H. (1975) Normal values in clinical chemistry. A guide to statistical analysis of laboratory data, Marcel Dekker, Inc., New York.

32. Krause, R. D. Anand, V. D. Gruemer, H. D. \& Willke, T. A (1975) Clin. Chem. 21, 321-324.

33. Robertson, E. A. \& Zweig, M. H. (1981) Clin. Chem. 27, 1569-1574.

34. Rea, J. N., Tyrer., Kasap, H. S. \& Beresford, S. A. A. (1973) Brit. J. Prev. Soc. Med. 27, 114-120.

35. Johnson Smith, N. (1977) J. Occup. Med. 19, 766-769.

36. Stewart, R. D., Stewart, R. S., Stamm, W. \& Seelen, R. P. (1976) J. Am. Med. Assoc. 235, 390-392.

37. Metz, Ch. E., Goodenough, D. J. \& Rossmann, K. (1973) Diagn. Radiol. 109, 297-304.

38. Oellerich, M. \& Haeckel, R. (1978) Med. Welt 29, 866-872.

39. Robertson, A., Zweig, M. H. \& van Steirteghem, A. C. (1983) Am. J. Clin. Pathol. 79, 78-86.

40. Haeckel, R., Riedel, H. \& Büttner, J. (1981) J. Clin. Chem. Clin. Biochem. 19, 173-176.

41. van der Helm, H. J. \& Hische, E. A. H. (1979) Clin. Chem. 25, 985-988.

42. Keller, H. \& Gessner, U. (1982), Schweiz. Med. Wochenschr. 112, 914-926.

Dr. G. Heinemann

Institut für Klinische Chemie

Deutsches Herzzentrum München

Lothstraße 11

D-8000 München 2 


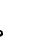

